\title{
Tratamento do Tromboembolismo Venoso em Pacientes com Câncer: Atualização quanto ao Papel dos Anticoagulantes Orais Diretos nesse Cenário
}

doi: https://doi.org/10.32635/2176-9745.RBC.2019v65n3.387

Venous Thromboembolism Treatment in Cancer Patients: Update of the Role of Direct Oral Anticoagulants in this Scenario

Tratamiento de Tromboembolismo Venoso en Pacientes con Cáncer: Actualización sobre el Papel de los

Anticoagulantes Orales Directos en este Escenario

\section{Marcos Jose Pereira Renni ${ }^{1}$; Tatiana Abelin Saldanha Marinho' ${ }^{2}$; Mirian Carvalho de Souza ${ }^{3}$}

\section{INTRODUÇÃO}

O câncer é amplamente conhecido por aumentar o risco de complicaçôes tromboembólicas. Esse risco está relacionado às características do próprio paciente, suas comorbidades e condiçóes clínicas, além de fatores relacionados ao tumor e ao momento do tratamento. A terapia anticoagulante deverá ser prescrita levando-se em consideraçáo o risco hemorrágico e de recorrência de trombose venosa profunda (TVP).

Estudos clínicos foram realizados para validar a utilização dos novos anticoagulantes orais diretos (DOAC) em pacientes com câncer associado à trombose (CAT). Entretanto, os riscos devem ser considerados com intuito de fornecer a terapia mais adequada e segura para cada paciente. Essas alternativas terapêuticas deverão ser divulgadas nos serviços de oncologia, facilitando assim sua utilização e maior benefício para os pacientes.

Estima-se que 20\% dos pacientes com câncer ativo irão desenvolver trombose no curso de seu tratamento ${ }^{1}$. Além disso, essa população em especial apresenta um risco de recorrência de TVP de cerca de três vezes comparada à população sem câncer². Por se tratar de pacientes, na maioria das vezes, debilitados, quer seja pela doença em si ou decorrente dos próprios tratamentos a que se submetem ao longo de sua evolução clínica, estão duas vezes mais sujeitos a eventos hemorrágicos na vigência das terapias anticoagulantes ${ }^{1,3}$.

O risco absoluto de desenvolver complicaçóes tromboembólicas depende do tipo de tumor, do estágio ou da extensão do câncer ou do tratamento com agentes antineoplásicos. Estima-se que pacientes oncológicos que desenvolveram TVP apresentem $94 \%$ de probabilidade de vir a falecer até seis meses após o episódio de TVP. Entende- -se, assim, que a TVP é considerada um marcador preditivo negativo de sobrevida nos pacientes oncológicos ${ }^{4,5}$.

Foram estimados, para cada ano do biênio 20182019, 324.580 casos novos de câncer entre os homens e 310.300 entre as mulheres no Brasil. Com base nessas informações, pode-se esperar um número significativo de eventos tromboembólicos entre pacientes oncológicos ${ }^{6}$.

Esse evento pode ocorrer em qualquer momento da evolução clínica do paciente, quer seja precedendo ao diagnóstico de câncer, no qual ele surge como um primeiro sinal ou sintoma do câncer, ou ainda durante as etapas de hospitalização ou tratamento. Esse risco diminui durante o período de remissão da doença. Entretanto, na doença metastática, o risco de TVP aumenta significativamente, bem como pode ser um primeiro sinal de recidiva da doença, quando o paciente está em controle clínico ${ }^{1}$.

Nesse cenário, ao prescrever um tratamento anticoagulante para o paciente com CAT, faz-se necessário individualizar esse paciente e avaliá-lo dentro de quatro aspectos clínico-epidemiológicos: i) fatores inerentes à história pregressa do paciente no que diz respeito a comorbidades, presença de veias varicosas, histórico de TVP ou de trombofilias; ii) características do tumor que o paciente apresenta; ou seja, o tipo e o grau histológico do tumor, o estádio em que se encontra e o momento do diagnóstico do câncer; iii) tratamento que está sendo aplicado: quimioterapias, cateteres venosos centrais, terapias antiangiogênicas, cirurgias, radioterapias, transfusóes sanguíneas, imobilidade e hospitalização; iv) biomarcadores: hematológicos, D-Dímero, proteína C-reativa, P-selectina, atividade de fator tecidual e protrombina fragmento $1+2^{1}$. A partir da avaliação do paciente nesses quatro aspectos, bem como da avaliação do risco hemorrágico e da concordância do paciente no que diz respeito à terapia 
prescrita, o tratamento anticoagulante será iniciado. Este deverá ser reavaliado levando-se em conta o seu dinamismo na evolução clínica do paciente em tratamento oncológico, que se encontra sujeito a múltiplas intercorrências e a mudanças na sua situação clínica.

\section{DESENVOLVIMENTO}

\section{QUE PRESCREVER PARA 0 PACIENTE COM CÂNCER ASSOCIADO À TROMBOSE?}

A avaliação do paciente com câncer associado a evento trombótico deve levar em consideração os fatores clinicoepidemiológicos descritos anteriormente, e estar de acordo com as condiçôes clínicas do paciente, as possíveis intervençóes que ele venha a se submeter, ao risco hemorrágico e partilhar com o paciente sua decisão, levando-se em consideração seu desejo.

Desde a aprovação dos DOAC, estudos de prática clínica, coortes observacionais, têm sido realizados descrevendo as experiências desses fármacos na população com câncer ${ }^{7}$. O foco principal está na eficácia, traduzida como recorrência de trombose na populaçáo, bem como na segurança; ou seja, na presença de sangramentos críticos ou fatais nessa população. Outro fator importante é a interação droga-droga com agentes quimioterápicos que podem alterar a farmacodinâmica dos DOAC aumentando o risco hemorrágico ou trombótico. Desse modo, o uso concomitante com algumas drogas, que são inibidores potentes ou indutores, deve ser evitado ${ }^{8}$.

Uma revisão sistemática da literatura descrevendo todos os estudos observacionais sobre esse tópico relatou que a maioria dos estudos usava rivaroxabana e heparinas de baixo peso molecular (HBPM) ${ }^{9}$. As duraçôes dos tratamentos com DOAC foram maiores comparadas às das HBPM, o que pode refletir as preferências dos pacientes por agentes orais e pelo custo. Quase todos os estudos relataram baixas taxas de recorrências nos grupos tratados com DOAC comparados aqueles que usaram HBPM. Com relação a sangramentos graves e menos importantes, foram encontrados dados heterogêneos. Entende-se que os estudos observacionais apresentam vieses que limitam algumas inferências sobre os dados descritos ${ }^{10}$.

Os pacientes relataram como mais importante a eficácia e a segurança dos anticoagulantes orais e preferem também sua administração por via oral, frente às injeçôes ${ }^{7}$. Sendo assim, a utilização dos DOAC como opção terapêutica permite uma maior comodidade no tratamento e maior adesão dos pacientes.

Nesse momento, apenas a rivaroxabana e edoxabana apresentam estudos pertinentes à população de pacientes oncológicos. As diferenças no mecanismo de ação da dabigatrana, que é um inibidor direto da trombina, não permitem fazer inferências ou comparaçóes aos outros anticoagulantes com mecanismo de ação anti-Xa.

A partir da avaliação individual do paciente e da decisão compartilhada, sugere-se DOAC específicos, edoxabana e rivaroxabana, para os pacientes com CAT. Entretanto, para aqueles pacientes com trombose aguda e alto risco hemorrágico, sugere-se HBPM, incluindo os pacientes com tumores luminais gastrointestinais intactos; ou seja, sem terem sido submetidos à ressecçáo, bem como naqueles com tumores do trato geniturinário, nefrostomias, úlceras duodenais ativas, esofagites ou colite 7 .

Outro ponto importante para refletir são aqueles pacientes com extremos de peso e redução acentuada da depuraçáo renal, em que se faz necessária cautela tanto para a prescrição de HBPM como também de DOAC.

\section{DOAC EM PACIENTES COM CÂNCER}

O Select-D trial foi um estudo cujo objetivo era a avaliaçáo de um inibidor oral do fator anti-Xa, no caso a rivaroxabana, como alternativa terapêutica para o tratamento de trombose em pacientes oncológicos. Foram recrutados pacientes em vários centros com câncer ativo e que tiveram tromboembolismo pulmonar e TVP. Em um braço dos estudos, foram alocados pacientes para tratamento com dalteparina, na dose de $200 \mathrm{UI} / \mathrm{Kg}$, diariamente, por um mês; e, a seguir, a dose foi ajustada para $150 \mathrm{UI} / \mathrm{Kg}$ para os meses seguintes. Em outro braço, foram alocados pacientes que foram tratados com rivaroxabana na dose de $15 \mathrm{mg}$, duas vezes ao dia, por 21 dias; e, a seguir, a dose foi ajustada para $20 \mathrm{mg}$, uma vez ao dia. O desfecho primário foi recorrência de trombose durante seis meses de acompanhamento ${ }^{11}$.

A segurança foi avaliada por sangramento maior e sangramento não significativo clinicamente relevante. $\mathrm{O}$ estudo foi realizado com um tamanho amostral de 400 pacientes. A taxa de recorrência de trombose em seis meses no braço dalteparina foi de $11 \%$ e no braço rivaroxabana, $4 \%$. A taxa cumulativa de sangramento e de sangramento maior foi de $4 \%$ para dalteparina e $6 \%$ para rivaroxabana. $\mathrm{O}$ estudo concluiu que a rivaroxabana foi associada a uma recorrência relativamente baixa de TVP, entretanto, com um aumento de sangramento do braço rivaroxabana ${ }^{11}$.

Outro estudo desenhado especificamente para avaliar anticoagulação em pacientes com câncer foi o Hokusai-cancer, cuja droga edoxabana, um inibidor direto do fator de coagulação ativado Xa, foi comparada à dalteparina. A proposta foi de tratamento por seis até 12 meses. No braço edoxabana, foi iniciada a anticoagulação com dalteparina subcutânea na dose de $200 \mathrm{UI} / \mathrm{kg}$, uma vez ao dia, por cinco dias; a seguir, edoxabana na dose de $60 \mathrm{mg} / \mathrm{dia}$. O braço dalteparina foi iniciado com a dose de $200 \mathrm{UI} / \mathrm{kg}$ por dia, por um mês; e, a seguir, a dose 
foi reduzida para $150 \mathrm{UI} / \mathrm{kg}$ por dia, até o término do tratamento. O resultado primário foi o desfecho composto por recorrência de trombose ou hemorragia grave durante os 12 meses de tratamento. O estudo avaliou os casos de baixo peso, clearence de creatinina entre $30-50 \mathrm{~mL} / \mathrm{min}^{12}$.

A escolha da terapia anticoagulante no paciente oncológico deverá ser individualizada e dinâmica, pois, a qualquer momento, podem ocorrer mudanças na evolução clínica do paciente, tanto em termos de complicaçôes, procedimentos, intervençóes, como em mudanças no plano terapêutico, necessitando a reavaliação do paciente. A interação droga-droga, funçôes renal e hepática, contagem de plaquetas e demais índices hematimétricos, risco de sangramentos maiores ou de recorrência de trombose devem ser considerados (Quadro 1).

A utilização de um algoritmo para nortear o diagnóstico e o tratamento de TVP nos setores de emergência e ambulatórios pode facilitar a prescrição mais adequada da terapia anticoagulante no paciente com CAT. Nesse sentido, desenvolveu-se, no Instituto Nacional de Câncer José Alencar Gomes da Silva (INCA), um algoritmo conforme apresentado na Figura 1. Uma conduta inicialmente adequada poderá promover maior eficácia e segurança para o paciente.

Quadro 1. Considerações clínicas durante a escolha da terapia anticoagulante para pacientes com câncer

\begin{tabular}{|c|c|c|}
\hline DROGA & AÇÃO & CONSIDERAR \\
\hline $\begin{array}{l}\text { Anticoagulantes } \\
\text { orais diretos }\end{array}$ & Ótimo & $\begin{array}{l}\text { Pacientes sem neoplasia gastrointestinal } \\
\text { Baixo risco de sangramento maior } \\
\text { Facilidade no tratamento oncológico } \\
\text { Baixa interação droga-droga }\end{array}$ \\
\hline $\begin{array}{l}\text { Anticoagulantes } \\
\text { orais diretos }\end{array}$ & Evitar & $\begin{array}{l}\text { Neoplasia gastrointestinal ativa } \\
\text { História de sangramento gastrointestinal prévio } \\
\text { Extremos de peso }(<50 \mathrm{Kg} \mathrm{e}>150 \mathrm{Kg})\end{array}$ \\
\hline $\begin{array}{l}\text { Heparinas de } \\
\text { baixo peso } \\
\text { molecular }\end{array}$ & Ótimo & $\begin{array}{l}\text { Terapias com náuseas e vômitos extremos } \\
\text { Alterações de absorção gastrointestinal } \\
\text { Interações droga-droga com anticoagulantes orais diretos e antivitamina K } \\
\text { Risco conhecido de sangramento } \\
\text { Câncer recorrente associado à trombose venosa profunda durante a anticoagulação }\end{array}$ \\
\hline $\begin{array}{l}\text { Heparinas de } \\
\text { baixo peso } \\
\text { molecular }\end{array}$ & Evitar & $\begin{array}{l}\text { Aversão do paciente à terapia parenteral } \\
\text { Insuficiência renal ou flutuação do status renal } \\
\text { Extremos de peso ( }<50 \mathrm{Kg} \mathrm{e}>150 \mathrm{Kg})\end{array}$ \\
\hline Antivitamina $\mathrm{K}$ & Ótimo & $\begin{array}{l}\text { Qualquer situação que se deva monitorar a anticoagulação (riscos de } \\
\text { sangramentos ou alterações metabólicas ou de absorção) } \\
\text { Insuficiência renal crônica avançada } \\
\text { Extremos de peso ( }<50 \mathrm{Kg} \text { e }>150 \mathrm{Kg} \text { ) }\end{array}$ \\
\hline Antivitamina $\mathrm{K}$ & Evitar & Falta de controle da anticoagulação-monitoramento \\
\hline
\end{tabular}

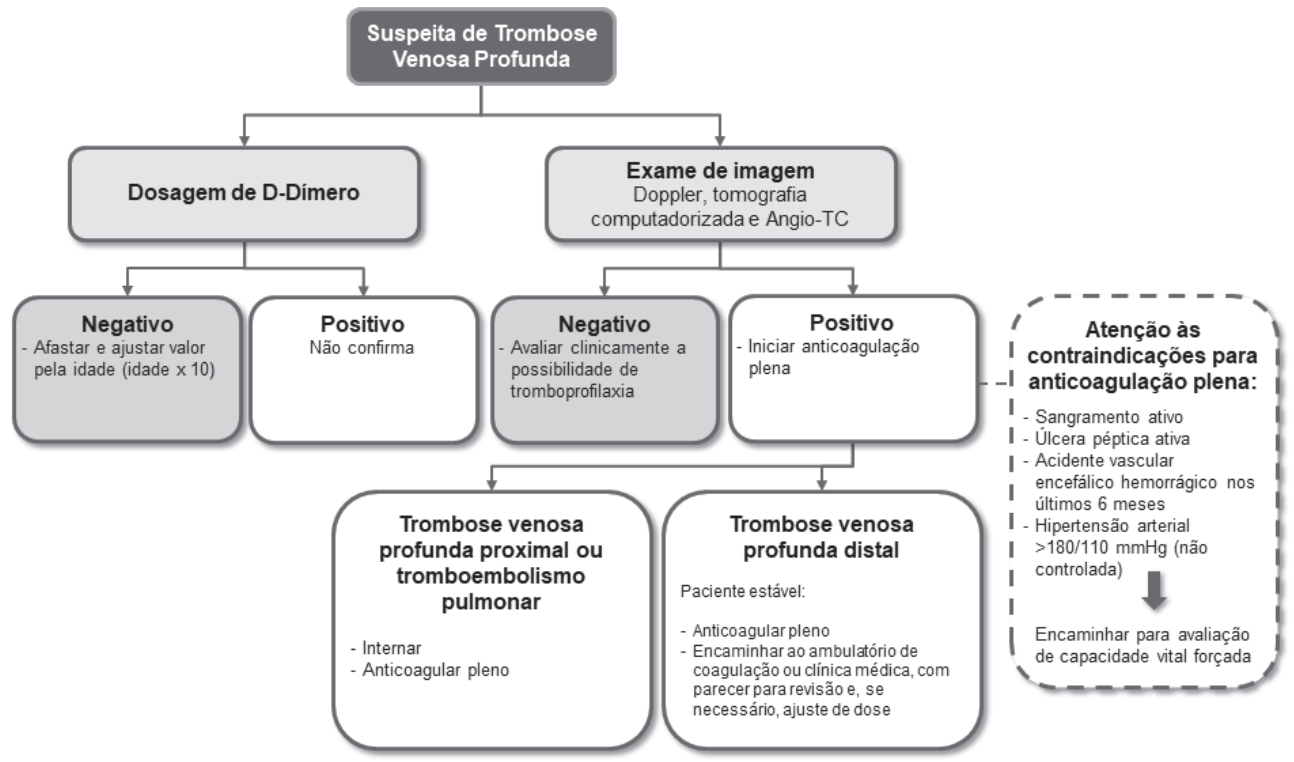

Figura 1. Algoritmo para diagnóstico e tratamento de TVP do INCA 


\section{CONCLUSÃO}

No paciente oncológico, os riscos de trombose e sangramentos são elevados. As condições clínicas de cada paciente devem sempre ser avaliadas, bem como se deve considerar o momento clínico em que ele se encontra.

A conduta terapêutica individualizada minimiza os riscos de recorrências de trombose e de hemorragias. Deve-se ter atenção aos pacientes com lesão no trato geniturinário, pelo grande risco hemorrágico e, finalmente, compartilhar com o paciente a decisão a ser tomada com relação à terapia anticoagulante.

\section{CONTRIBUIÇÕES}

Marcos Jose Pereira Renni e Tatiana Abelin Saldanha Marinho participaram da concepção e planejamento do estudo, análise e interpretação dos dados, redação e revisão crítica do manuscrito. Mirian Carvalho de Souza participou da redação e revisão crítica do manuscrito. Todos os autores aprovaram a versão final a ser publicada.

\section{DECLARAÇÃO DE CONFLITO DE INTERESSE}

Nada a declarar.

\section{FONTES DE FINANCIAMENTO}

Não há.

\section{REFERÊNCIAS}

1. Ay C, Pabinger I, Cohen AT. Cancer-associated venous thromboembolism: burden, mechanisms, and management. Thromb Haemost. 2017 Jan 26;117(2):21930. doi: http://dx.doi.org/10.1160/TH16-08-0615

2. Prandoni P, Falanga A, Piccioli A. Cancer and venous thromboembolism. Lancet Oncol. 2005;6(6):401-10. doi: http://dx.doi.org/10.1016/S1470-2045(05)702072. https://doi.org/10.1016/S1470-2045(05)70207-2

3. Prandoni P, Lensing AW, Piccioli A, et al. Recurrent venous thromboembolism and bleeding complications during anticoagulant treatment in patients with cancer and venous thrombosis. Blood. 2002 Nov 15;100(10):3484-8. doi: https://doi.org/10.1182/ blood-2002-01-0108

4. Parkin M, Pisani P, Ferlay J. Global cancer statistics. CA Cancer J Clin. 1999;49(1):33-64. doi: http://dx.doi. org/10.3322/canjclin.49.1.33.

5. Renni MJP, Cerqueira MH, Trugilho IA, et al. Mecanismos do tromboembolismo venoso no câncer: uma revisão da literatura. J Vasc Bras. 2017;16(4):308313. doi: http://dx.doi.org/10.1590/1677-5449.007817
6. Instituto Nacional de Câncer José Alencar Gomes da Silva. Estimativa 2018: incidência de câncer no Brasil [Internet]. Rio de Janeiro: INCA; 2017. [acesso 2018 dez. 15]. Disponível em: https://www.inca.gov.br/sites/ ufu.sti.inca.local/files//media/document//estimativaincidencia-de-cancer-no-brasil-2018.pdf

7. Khorana AA, Noble S, Lee Ayy, et al. Role of direct oral anticoagulants in the treatment of cancer-associated venous thromboembolism: guidance from the SSC of the ISTH. J Thromb Haemost. 2018 Sep;16(9):1891-94. doi: https://doi.org/10.1111/jth.14219

8. Lee AY, Peterson EA. Treatment of cancer-associated thrombosis. Blood. 2013;122(14):2310-17. doi: https:// doi.org/10.1182/blood-2013-04-460162

9. Li A, Garcia DA, Lyman GH, et al. Direct oral anticoagulant (DOAC) versus low-molecular-weight heparin (LMWH) for treatment of cancer associated thrombosis (CAT): a systematic review and metaanalysis. Thromb Res. 2019;173:158-163. doi: https:// doi.org/10.1016/j.thromres.2018.02.144

10. Lee Ayy, Kamphuisen PW, Meyer G, et al. Tinzaparin vs warfarin for treatment of acute venous thromboembolism in patients with active cancer: a randomized clinical trial. JAMA. 2015;314(7): 677-86. doi: https://doi. org/10.1001/jama.2015.9243

11. Young AM, Marshall A, Thirlwall J, et al. Comparison of an oral factor xa inhibitor with low molecular weight heparin in patients with cancer with venous thromboembolism: results of a randomized trial (SELECT-D). J Clin Oncol. 2018;36(20):2017-23. doi: https://doi.org/10.1200/JCO.2018.78.8034

12. Raskob GE, van Es N, Segers A, et al. Edoxaban for venous thromboembolism in patients with cancer: results from a non-inferiority subgroup analysis of the HokusaiVTE randomised, double-blind, double-dummy trial. Lancet Haematol. 2016 Aug;3(8):e379-87. doi: https:// doi.org/10.1016/S2352-3026(16)30057-6

Recebido em 8/8/2019 Aprovado em 10/10/2019 